\title{
Endoscopic findings of gastric lesions in patients with eosinophilic gastrointestinal disorders
}

\section{(C) (i) $\odot$}

\section{Authors}

Yasuhiro Fujiwara ${ }^{1}$, Kojiro Tanoue ${ }^{1}$, Akira Higashimori ${ }^{1}$, Yu Nishida ${ }^{1}$, Masatsugu Maruyama ${ }^{1}$, Shigehiro Itani ${ }^{1}$, Masaki Ominami ${ }^{1}$, Yuji Nadatani ${ }^{1,2}$, Shusei Fukunaga ${ }^{1}$, Koji Otani ${ }^{1}$, Shuhei Hosomi ${ }^{1}$, Fumio Tanaka ${ }^{1}$, Noriko Kamata ${ }^{1}$, Yasuaki Nagami ${ }^{1}$, Koichi Taira ${ }^{1}$, Hirohisa Machida ${ }^{3}$, Tetsuya Tanigawa ${ }^{4}$, Toshio Watanabe ${ }^{1}$, Masahiko Ohsawa ${ }^{5}$, F-Study group

Institutions

1 Department of Gastroenterology, Osaka City University Graduate School of Medicine, Osaka, Japan

2 Department of Premier Preventive Medicine, Osaka City University Graduate School of Medicine, Osaka, Japan

3 Department of Internal Medicine, Machida Gastrointestinal Hospital, Osaka, Japan

4 Department of Gastroenterology, Osaka City Juso Hospital, Osaka, Japan

5 Department of Diagnostic Pathology, Osaka City University Graduate School of Medicine, Osaka, Japan

submitted 17.6.2020

accepted after revision 3.9.2020

\section{Bibliography}

Endoscopy International Open 2020; 08: E1817-E1825

DOI 10.1055/a-1268-7312

ISSN 2364-3722

(C) 2020. The Author(s).

This is an open access article published by Thieme under the terms of the Creative Commons Attribution-NonDerivative-NonCommercial License, permitting copying and reproduction so long as the original work is given appropriate credit. Contents may not be used for commecial purposes, or adapted, remixed, transformed or built upon. (https://creativecommons.org/licenses/by-nc-nd/4.0/)

\section{Corresponding author}

Dr. Yasuhiro Fujiwara, Department of Gastroenterology, Osaka City University Graduate School of Medicine, 1-4-3

Asahimachi, Abenoku, Osaka 545-8585, Japan

Fax: +81-6-6645-3813

yasu@med.osaka-cu.ac.jp

\section{ABSTRACT}

Background and study aims Eosinophilic gastrointestinal disorders are classified into eosinophilic esophagitis, eosinophilic gastritis, eosinophilic gastroenteritis, and eosinophilic colitis according to the site of eosinophilic infiltration. Although well established in eosinophilic esophagitis, endoscopic findings in eosinophilic gastritis and eosinophilic gastroenteritis with regard to gastric lesions have not been clearly described. The aim of this study was to identify endoscopic findings of gastric lesions associated with eosinophilic gastrointestinal disorders.

Patients and methods Out of 278 patients with eosinophilic gastrointestinal disorders, 18 had eosinophilic gastritis or eosinophilic gastroenteritis confirmed by biopsy; their endoscopic images were analyzed retrospectively. The association between endoscopic findings and number of eosinophils in the gastric mucosa was investigated.

Results Erythema was most frequently observed (72\%), followed by ulcers (39\%), discoloration (33\%), erosions (28\%), nodularity (28\%), and polyps (28\%). There were several unique endoscopic findings such as submucosal tumorlike deep large ulcers in three patients, antral Penthorumlike appearances (small nodules radially lined toward the pyloric ring) in three patients, "muskmelon-like appearances" (discolored mucosa-composed mesh pattern) in three patients, multiple white granular elevations in two patients, cracks (appearance of furrows similar to those in eosinophilic esophagitis) in five patients, and antral rings in one patient. No significant association was observed between endoscopic findings and number of gastric eosinophils.

Conclusions Several unique endoscopic findings of gastric lesions were observed in patients with eosinophilic gastritis or eosinophilic gastroenteritis. Submucosal tumor-like ulcers, antral Penthorum-like appearances, muskmelon-like appearances, and cracks might be associated with eosinophilic gastrointestinal disorders. 


\section{Introduction}

Eosinophilic gastrointestinal disorders (EGIDs) are chronic allergic diseases of the gastrointestinal (gastrointestinal) tract and are classified into eosinophilic esophagitis (EoE), eosinophilic gastritis (EG), eosinophilic gastroenteritis (EGE), and eosinophilic colitis (EC) according to the site of eosinophilic infiltration $[1,2]$. In the first Japanese study on EGIDs, 26 patients with EoE and 144 with EGE between 2004 and 2009 were included; it suggested that EoE is less prevalent than non-EoE EGIDs, such as EG, EGE, and EC [3]. However, since then, the prevalence of EoE has been increasing [4,5], and the prevalence of non-EoE EGIDs is currently considered to be low in Japan. Although the epidemiology of non-EoE EGIDs is unclear, a recent US study that used the insurance database reported that EG, EGE, and EC are rare and have standardized prevalence of 6.3/100,000, $8.4 / 100,000$, and $3.3 / 100,000$, respectively [6].

Diagnosis of EGIDs is based on the presence of gastrointestinal symptoms and dense eosinophilic infiltration in the gastrointestinal tract [1, 2,7-10]. However, endoscopic findings play a key role in the initial diagnosis of patients refractory to standard treatment or those suspected to have EGIDs. Although endoscopic findings in EoE such as edema, rings, white exudates, furrows, and strictures are well established [8] and a grade system [11] is widely used in research, endoscopic findings of gastric lesions in EGIDs have not been clearly described due to the rarity of EG and EGE and due to the non-specificity of the endoscopic findings presented in earlier reports. In this study, we assessed patients with EGIDs in our department and collected images of gastric lesions in patients with EG or EGE to identify the associated endoscopic findings.

\section{Patients and methods}

\section{Study subjects}

A total of 298 patients suspected to have EGIDs were referred to our department between January 2009 and January 2020. We excluded eight patients without tissue eosinophilia and 12 with disorders of other etiologies. We assessed 278 patients with EGIDs and biopsy confirmed gastric eosinophilia in 19 patients. As detailed endoscopic images for one patient were missing, we assessed 18 patients with gastric lesions. We collected clinical data such as age, sex, height, body weight, smoking and alcohol drinking status, presence of allergic diseases, symptoms, disease duration, and blood test parameters including peripheral eosinophil count, hemoglobin level, and albumin level from medical charts. This study was approved by the ethics committee of Osaka City University (approval number: 4141) and conducted in accordance with the ethical principles of the Declaration of Helsinki. Written informed consent was obtained from all participants before endoscopic examination. All patients were provided the opportunity to opt out from this study.

\section{Diagnosis of non-EoE EGIDs (EG, EGE, and EC)}

Diagnosis of non-EoE EGIDs was based on the following guidelines from the Japanese Ministry of Health, Labor and Welfare $[1,12]: 1$. presence of symptoms such as abdominal pain, diar- rhea, and vomiting; 2 . mucosal eosinophilic infiltration of $\geq 20$ eosinophils per high-power field (eos/hpf) on biopsy of the stomach, small intestine, or large intestine; 3. exclusion of other diseases such as inflammatory bowel disease, parasitic infections, and systemic diseases; and 4. presence of ascites and high number of eosinophils in ascites. Patients with any gastrointestinal symptom and $\geq 20$ eos/hpf on gastric biopsy were diagnosed with EG or EGE, regardless of the presence of esophageal eosinophilia.

\section{Endoscopic examination and biopsy protocol}

Upper gastrointestinal endoscopy was performed using EGL600-LR7 (Fujifilm, Tokyo, Japan) or Q240, Q260, H260, H290, or H290-Z (Olympus, Tokyo, Japan) endoscopes. Midazolam was used for sedation in some patients. After careful examination of the esophagus, stomach, and duodenum, biopsies were performed using single-use Radial Jaw 4 (Boston, Scientific Japan, Tokyo, Japan). Targeted biopsies were performed when there were abnormal endoscopic findings, and random biopsies were performed at the discretion of each endoscopist.

\section{Histological assessment}

The biopsy samples were fixed in formalin, embedded in paraffin, cut into $4 \mu \mathrm{m}$ sections, and stained with hematoxylin-eosin. Intraepithelial eosinophils were counted using $\times 40$ and $\times 10$ objective lenses (BX50 optical microscope, Olympus, Tokyo, Japan). For each specimen, peak eosinophil counts of easily recognizable areas of eosinophilia were evaluated in a low-power field and expressed as the maximum number in a high-power field $\left(0.24 \mathrm{~mm}^{2}\right)$. Diagnosis was made by experienced pathologists.

\section{Endoscopic assessment}

All pre-treatment endoscopic images of the stomach were collected, assessed, and categorized into ulcer, erosion, erythema, nodularity, discoloration, or others. Each endoscopic finding was defined and described as follows:

\section{Ulcers and erosions}

The number, size, shape, depth, and location of ulcers and erosions were evaluated. Ulcers were subdivided into large ulcers $(\geq 2 \mathrm{~cm}$ in diameter; $>$ Fig. $1 \mathrm{a})$ and small ulcers $(<2 \mathrm{~cm}$ in diam-

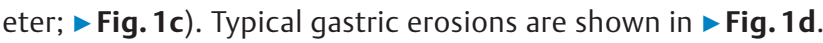

\section{Erythema}

Erythema was categorized according to the 2013 Kyoto gastritis classification of the Japanese Gastroenterological Endoscopy Society, which has listed 19 endoscopic findings related to gastritis [13-15]. Among these findings, diffuse redness, map-like redness, red streaks, patchy redness, and spotty redness were proposed as characteristic of erythema. In addition, localized diffuse redness, defined as strong redness in a restricted area, has been added in this study ( $\triangleright$ Fig. $1 \mathbf{e}$ ). 

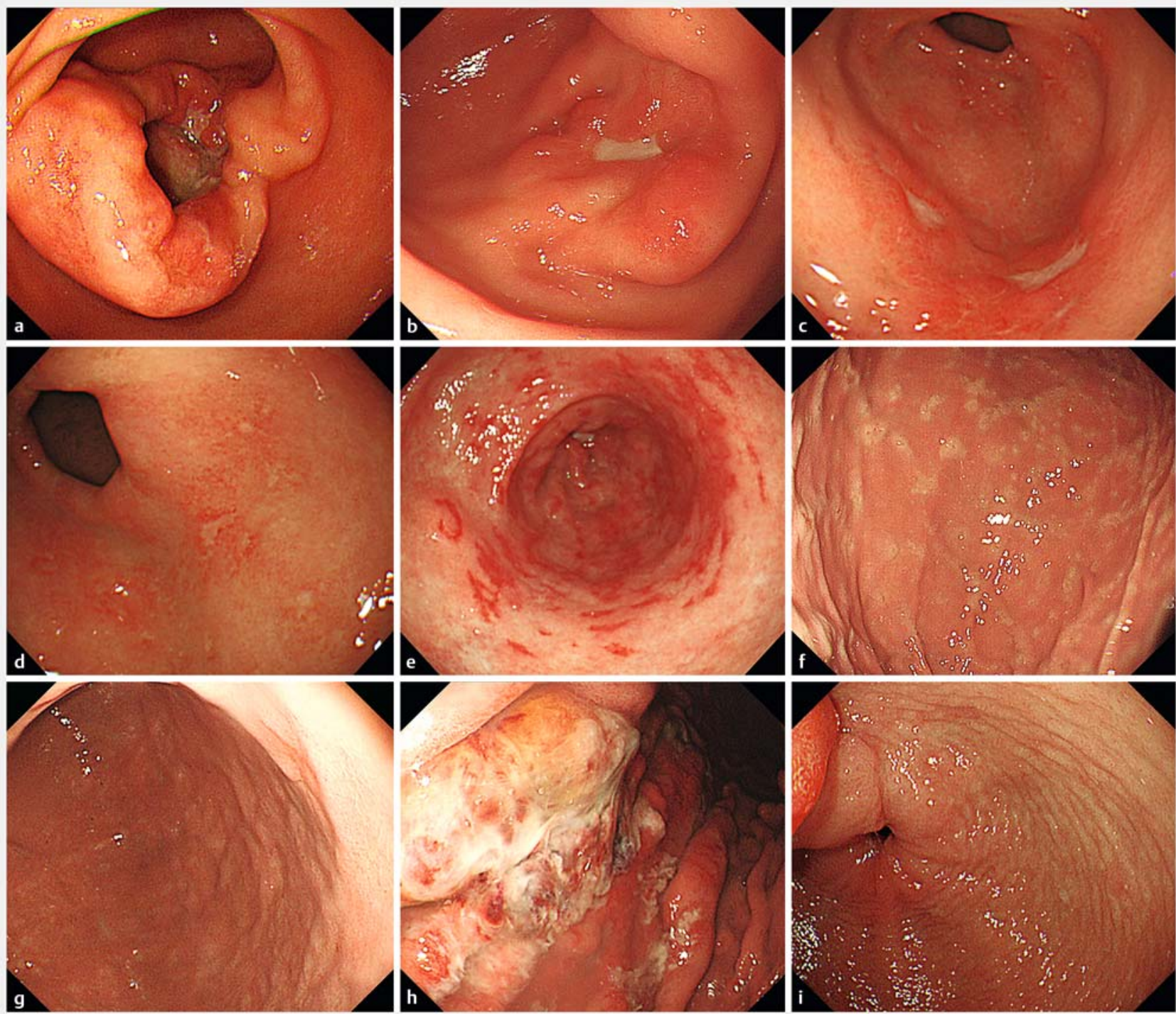

- Fig. 1 Several endoscopic findings of gastric lesions in patients with eosinophilic gastrointestinal disorders. a Submucosal tumor-like deep large ulcer. b Ulcer size and marginal elevation are reduced, and reddish regenerative mucosa is observed after food elimination therapy. c Multiple shallow small ulcers. d Multiple erosions. e Localized diffuse redness. $\mathbf{f}$ Multiple white granular elevations. $\mathbf{g}$ Patchy discoloration. h Marked white coats. i Antral rings.

\section{Nodularity}

Nodularity was categorized into two types. Multiple white granular elevation includes small whitish nodules with a granular pattern on the surface ( $\mathbf{F i g . 1 f}$ ). Antral Penthorum-like appearance includes small nodules radially lined toward the pyloric ring on the antrum ( $>$ Fig. 2 ) and some nodules with erosion or redness on the top, similar to the raised erosion described in

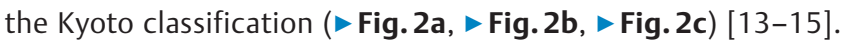
Penthorum chinense Pursh, a rooted vascular plant distributed in eastern Asia, is frequently found in the muddy wetland, riparian food plains and fallow paddy fields in Japan and resembles the feet of octopus.

\section{Discoloration}

Discoloration was classified into two types: multiple patchy type and muskmelon-like appearances. The multiple patchy type is defined as several small patchy discolorations not con-

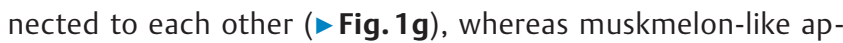
pearance is defined as discolored mucosa-composed mesh patterns ( $\mathbf{F i g} . \mathbf{3}$ ).

\section{Cracks}

Cracks were defined as crackled-like appearance of the gastric mucosa that was endoscopically detected using multiple depressed lines [16]. The location of cracks was evaluated, and the crack patterns were divided into linear type ( $\triangleright$ Fig. $4 \mathbf{b}$ and $\triangleright$ Fig. 4d) and mesh-like type ( Fig.4a, $>$ Fig. 4c). 


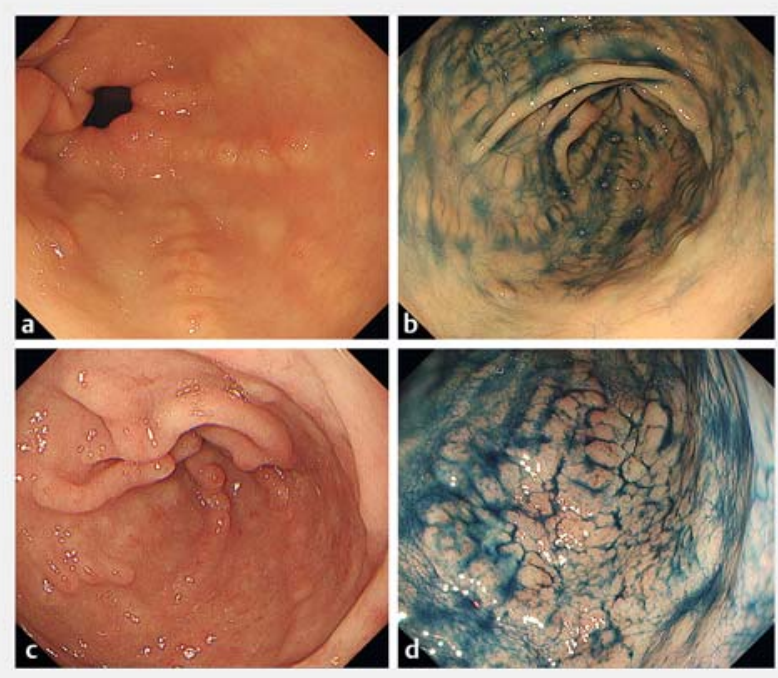

Fig. 2 Antral Penthorum-like appearances. a Small nodules lined radially toward to pyloric ring, hence the name antral Penthorumlike appearance. b Indigocarmine contrast image. c, $\mathbf{d}$ Other cases of antral Penthorum-like appearance.

\section{Other findings}

We assessed other findings such as polyps including hyperplastic polyps and fundic gland-type polyps, white coat ( $\mathbf{F i g . 1} \mathbf{~ h}$ ), and antral rings, revealing ring-shaped lines around the pylorus ( Fig. 1i).

\section{Efficacy of treatment on endoscopic findings}

Pretreatment and post-treatment endoscopic images were evaluated and compared. Efficacy was categorized into complete remission (healed mucosa or disappearance), remission (decreased size or shape of the lesions or relatively normalized color of the mucosa), and no change/worsening.

\section{Helicobacter pylori infection status}

H. pylori-positive infection was defined as the presence of $H$. pylori histologically and/or positive results of other diagnostic modalities such as serum antibody and rapid urease test. H. pylori-negative infection was defined as the absence of $\mathrm{H}$. pylori histologically and absence of atrophic gastritis on endoscopy. H. pylori infection status was classified as positive, negative, or post-eradication.

\section{Statistical analysis}

Data were expressed as mean \pm standard deviation, median and interquartile range (IQR), or number (frequency). Data were analyzed using one-way analysis of variance, followed by Dunnett's test for statistical comparisons. The level of statistical significance was $P<0.05$. All analyses were performed with EZR (Saitama Medical Center, Jichi Medical University, Saitama, Japan), which is a graphical user interface for $\mathrm{R}$ (The R Foundation for Statistical Computing, Vienna, Austria). More precisely, it is a modified version of $\mathrm{R}$ commander designed to add statistical function frequently used in biostatistics [17].

\section{Results}

\section{Study subjects}

Clinical characteristics of the study participants are shown in - Table 1. Of the 18 patients assessed, 16 were categorized as EGE and two as EG. Most patients ( $89 \%$ ) had allergic diseases, including bronchial asthma in seven patients, food allergy in five, allergic rhinitis in four, and drug allergy in three. The patients had varied symptoms because 16 patients had eosinophilia in different parts of the gastrointestinal tract: the esophagus $(n=10)$, small intestine $(n=10)$, and colon $(n=2)$. The range of gastric eosinophil count was 20-280 eos/hpf, and the mean peak number was $81.9 \mathrm{eos} / \mathrm{hpf}$.
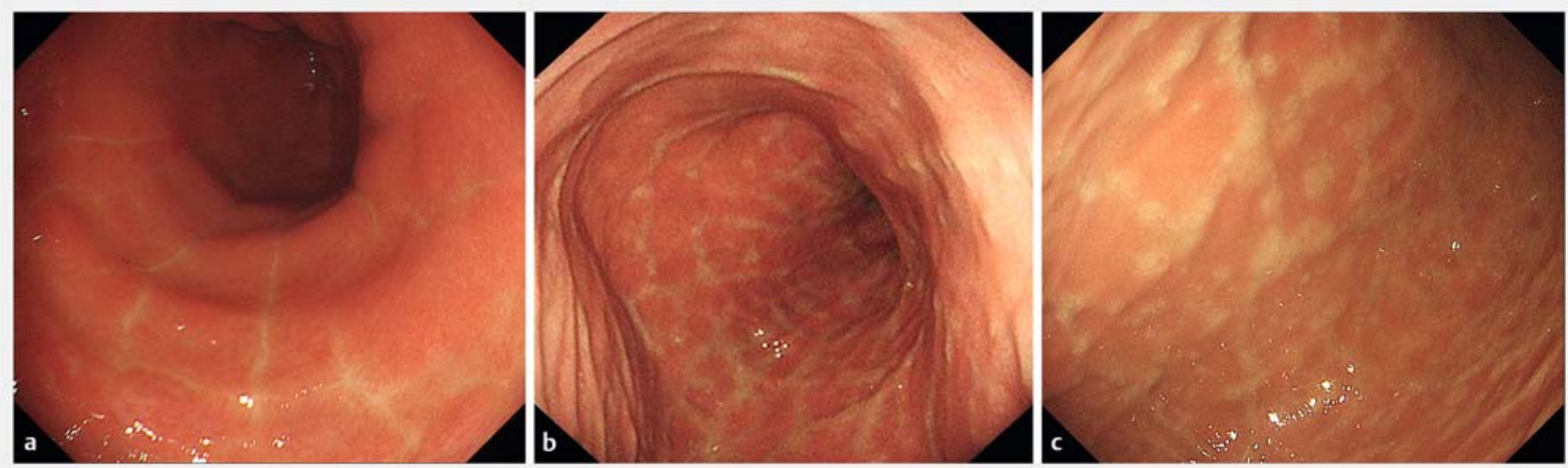

- Fig. 3 Muskmelon-like appearances. a, b, c Three cases of discolored mucosa-composed mesh pattern referred to as muskmelon-like appearances are shown. 


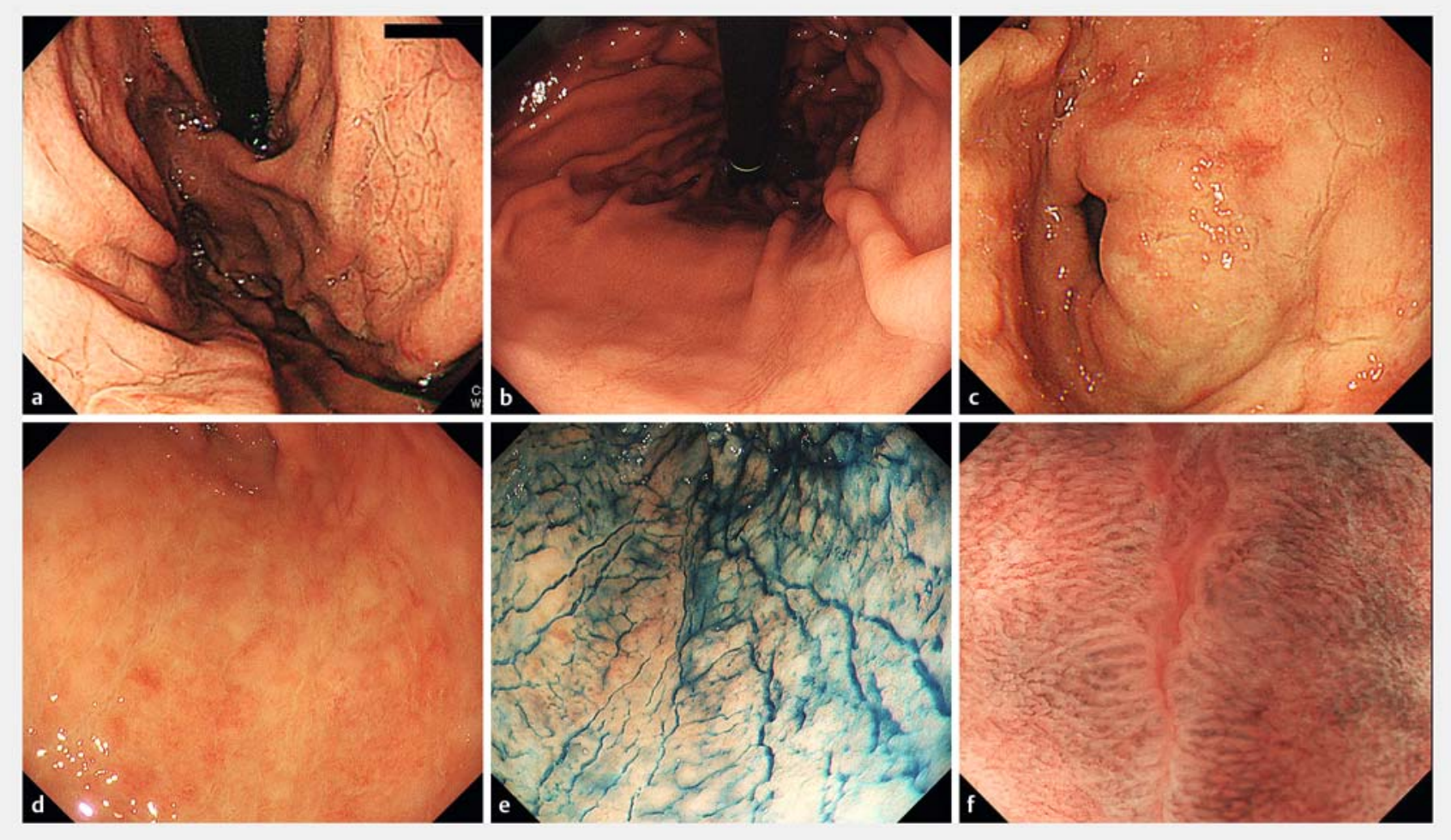

- Fig. 4 Cracks of several patterns observed in different parts of the stomach. a Cracks on the upper gastric body. $\mathbf{b}$ Cracks on the lesser curvature of the gastric body showing the linear furrows of eosinophilic esophagitis. c Cracks on the antrum near the pylorus. $\mathbf{d}$, e, $\mathbf{f}$ Cracks on the greater curvature of the antrum. $\mathbf{d}$ White-light image. e Indigocarmine contrast image. $\mathbf{f}$ Magnifying narrow-band image.

\section{Endoscopic findings}

The gastric lesions are summarized in $>$ Table 2 . Erythema was most frequently observed (72\%), followed by ulcers (39\%), discoloration (33\%), erosions ( $28 \%)$, nodularity ( $28 \%)$, and polyps (28\%).

Of the seven ulcers observed, five were large ulcers ( 3 in the antrum and 2 in the gastric body) and two were small ulcers. A typical large ulcer is shown in $\mathbf{F i g . 1}$ a and $>$ Fig. 1b. A submucosal tumor (SMT)-like deep large ulcer was observed on the greater curvature of the antrum ( $\triangleright$ Fig. 1a). After food elimination therapy, symptoms improved, ulcer size and marginal elevation reduced, and reddish regenerative mucosa was observed ( $\triangleright$ Fig. 1b). Three of the five large ulcers were SMT-like deep ulcers, and the other two large ulcers had no marginal elevation and were located in the pre-pylorus. Two patients had multiple shallow small ulcers in the antrum ( $\triangleright$ Fig. 1c), and five had multiple erosions located in the antrum (1 patient) and the gastric body (4 patients). Several types of erythema were observed such as patchy redness in 10 patients, red streaks in three, localized diffuse redness in two, and spotty redness in 13 (72\%). Two patients had multiple white granular elevations on the gastric body and three had small nodules radially lined toward the pyloric ring on the antrum, referred to as a antral Penthorumlike appearance (two had nodules with raised erosion ( $\triangleright$ Fig. 2a, > Fig. 2b, > Fig. 2c) and one had a relatively flat elevation ( $\triangleright$ Fig. 2d). Discoloration was found in six patients (33\%): three with multiple patchy type and three with muskmelon-like ap-


had cracks, and no patients received proton pump inhibitors (PPIs). Cracks were located in the antrum (two patients), the body (two patients), and both the antrum and the body (one patients). Of the three patients with cracks in the antrum, two had a mesh-like pattern ( $\mathbf{F i g . 4 c}$ ) and one had a linear pattern ( Fig.4d, $>$ Fig.4e, $>$ Fig.4f). One patient had a mesh-like pattern in the body ( $>$ Fig.4a) and linear furrows, which were similar to endoscopic findings in EoE found on the lesser curvature of the gastric body in three patients with cracks ( $>$ Fig. 4b). Five polyps consisting of two hyperplastic polyps and three fundic gland-type polyps were observed, and one patient had multiple hyperplastic polyps. Other findings included a marked white coat in one patient and antrum rings in one patient. Of the two patients with $H$. pylori-positive infection, one had small ulcers with red streak and the other had antrum rings with patchy redness. Of the three patients with post-eradication, one had erosion and fundic gland-type polyps; one had muskmelonlike appearance, hyperplastic polyp, and cracks; and one had multiple hyperplastic polyps with white coat and patchy redness.

Of these endoscopic findings of gastric lesions in patients with EG or EGE, SMT-like deep ulcers, antral Penthorum-like appearance, muskmelon-like appearance, multiple white granular elevations, cracks, and antral rings were considered relatively unique endoscopic findings. 
- Table 1 Clinical characteristics of the study subjects.

\begin{tabular}{|c|c|}
\hline \multicolumn{2}{|l|}{ Characteristic } \\
\hline Age (years) & $46.7 \pm 18.9$ \\
\hline Sex (male/female) & $5 / 13$ \\
\hline Body mass index $\left(\mathrm{kg} / \mathrm{m}^{2}\right)$ & $21.7 \pm 5.1$ \\
\hline Smoking habits (\%) & $1(1 \%)$ \\
\hline Drinking habits (\%) & $5(28 \%)$ \\
\hline Allergic diseases (\%) & $16(89 \%)$ \\
\hline \multicolumn{2}{|l|}{ Symptoms (\%) } \\
\hline - Epigastric pain & $7(39 \%)$ \\
\hline - Nausea/vomiting & $4(22 \%)$ \\
\hline - Dysphagia & $3(17 \%)$ \\
\hline - Edema & $2(11 \%)$ \\
\hline - Gastric discomfort & $1(6 \%)$ \\
\hline - Diarrhea & $1(6 \%)$ \\
\hline Disease duration & 1 month- 10 years \\
\hline \multicolumn{2}{|l|}{ Blood examination } \\
\hline - Peripheral eosinophilia ( $\geq 500 / f l)$ & $14(78 \%)$ \\
\hline - $\mathrm{Hb}(\mathrm{g} / \mathrm{dL})$ & $12.7 \pm 1.7$ \\
\hline . Alb (mg/L) & $3.6 \pm 0.7$ \\
\hline Eosinophils of the stomach (eos/hpf) & $48(27-110.5)$ \\
\hline \multicolumn{2}{|l|}{ H. pylori infection } \\
\hline - Positive & $2(11 \%)$ \\
\hline - Negative & $13(72 \%)$ \\
\hline - Post-eradication & $3(17 \%)$ \\
\hline \multicolumn{2}{|c|}{$\begin{array}{l}\text { Data were mean } \pm S D \text {, median (IQR), or number (frequency). } \\
\text { Hb, hemoglobin; Alb, albumin; SD, standard deviation; IQR, interquartile } \\
\text { range. }\end{array}$} \\
\hline
\end{tabular}

\section{Association between endoscopic findings and gastric eosinophilia}

In this study, 73 biopsies were performed and the mean number of biopsies per patient was 4.1. Fig. 5 shows a plot of number of infiltrated eosinophils in the gastric mucosa against endoscopic findings. There were no significant differences in the number of infiltrated eosinophils between the endoscopic findings. The median (IQR) number of eosinophils was $36.0 \mathrm{eos} / \mathrm{hpf}$ (30.0-70.0 eos/hpf) in ulcers, 62.5 eos/hpf (27.5-100.0 eos/ hpf) in erosions, $20.0 \mathrm{eos} / \mathrm{hpf}(11.5-37.5 \mathrm{eos} / \mathrm{hpf}$ ) in erythemas, $22.0 \mathrm{eos} / \mathrm{hpf}$ (2.5-39.5 eos/hpf) in nodularities, $118.0 \mathrm{eos} / \mathrm{hpf}$ (70.0-141.0 eos/hpf) in discolorations, $62.5 \mathrm{eos} / \mathrm{hpf}$ (27.5$100.0 \mathrm{eos} / \mathrm{hpf}$ ) in cracks, and $27.0 \mathrm{eos} / \mathrm{hpf}$ (18.5-53.8 eos/hpf) in normal background mucosa. The positive rate of gastric eosinophilia was $88.2 \%$ in ulcers, $100 \%$ in erosions, $54.5 \%$ in erythemas, $63.6 \%$ in nodularities, $100 \%$ in discolorations, $33 \%$ in
- Table 2 Endoscopic findings of gastric lesions in patients with EGIDs.

\begin{tabular}{|c|c|c|}
\hline Lesions & Types & Number (\%) \\
\hline \multirow[t]{3}{*}{ Ulcers } & & $7(39 \%)$ \\
\hline & Large ulcers & $5(28 \%)$ \\
\hline & Small ulcers & $2(11 \%)$ \\
\hline Erosions & & $5(28 \%)$ \\
\hline \multirow[t]{5}{*}{ Erythema } & & $13(72 \%)$ \\
\hline & Patchy redness & $10(56 \%)$ \\
\hline & Red streak & $3(17 \%)$ \\
\hline & Localized diffuse redness & $2(11 \%)$ \\
\hline & Spotty redness & $1(6 \%)$ \\
\hline \multirow[t]{3}{*}{ Nodularity } & & $5(28 \%)$ \\
\hline & Multiple white granular elevation & $2(11 \%)$ \\
\hline & Antral Penthorum-like appearance & $3(17 \%)$ \\
\hline \multirow[t]{3}{*}{ Discoloration } & & $6(33 \%)$ \\
\hline & Multiple patchy discoloration & $3(17 \%)$ \\
\hline & Muskmelon-like appearance & $3(17 \%)$ \\
\hline Cracks & & $5(28 \%)$ \\
\hline \multirow[t]{4}{*}{ Others } & & $5(28 \%)^{1}$ \\
\hline & Polyps & $5(28 \%)$ \\
\hline & White coat & $1(6 \%)$ \\
\hline & Antral rings & $1(6 \%)$ \\
\hline $\begin{array}{l}\text { EGID, eosinophi } \\
{ }^{1} \text { There were tw }\end{array}$ & $\begin{array}{l}\text { gastrointestinal disorder. } \\
\text { dings in two patients. }\end{array}$ & \\
\hline
\end{tabular}

cracks, and $75 \%$ in normal mucosa. Fifty-three target biopsies and 20 random biopsies were performed, and there was no significant difference in the positive rate of gastric eosinophilia between target biopsies (75.1\%) and random biopsies (75.0\%). There was also no significant difference in the number of gastric eosinophils between the biopsy sites as shown below: 27.5 eos/ hpf (13.5-52.0 eos/hpf) in the antrum and $30.0 \mathrm{eos} / \mathrm{hpf}$ (20.0$70.0 \mathrm{eos} / \mathrm{hpf}$ ) in the gastric body.

\section{Changes in endoscopic findings at post-treatment}

Eleven patients received systemic steroid therapy (including 7 patients received both steroid and PPIs or potassium-competitive acid blockers [P-CAB]), two topical steroid therapy, four PPIs or P-CAB, and one food elimination therapy. Post-treatment endoscopic examination in four patients was not performed. Of the patients with ulcer, four achieved complete remission, two achieved remission, and one had no changes. Among the four patients with erosions, two achieved complete remission and two had no changes. Of the patients with erythema, eight of nine cases with patchy erythema, one of two red streaks, one of two localized diffuse redness, and one spotty redness achieved complete remission or remission. Patients 


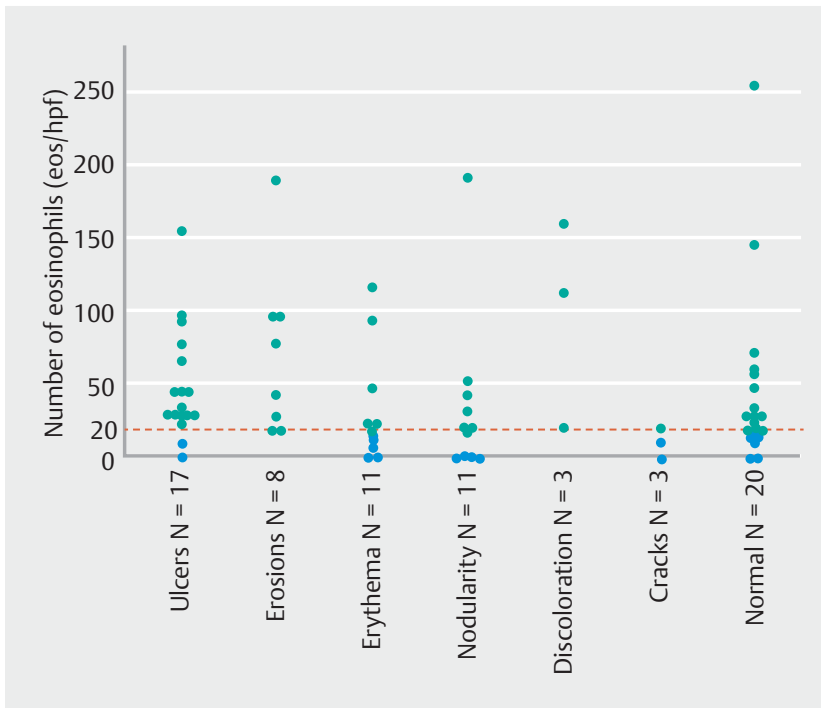

Fig. 5 Association between endoscopic findings and gastric eosinophilia. The peak number of eosinophils in the gastric mucosa in each specimen was plotted against the endoscopic findings. The green dots represent gastric eosinophilia ( $\geq 20 \mathrm{eos} / \mathrm{hpf}$ ) and the blue dots represent non-eosinophilia (<20 eos/hpf).

with multiple white granular elevation achieved complete remission; however, two patients with antral Penthorum-like appearances had no changes. Three patients with multiple patchy discoloration achieved complete remission, whereas two patients with muskmelon-like appearances achieved remission, but had not completely recovered. All patients with cracks had no changes at post-treatment.

\section{Discussion}

Data on the endoscopic findings of gastric lesions in patients with EGIDs are limited, and mostly derived from case reports. This study is the first to report the various endoscopic findings of gastric lesions in patients with EGIDs in detail. Erythemas were the most common finding observed ( $72 \%)$, followed by ulcers $(39 \%)$, discolorations (33\%), erosions ( $28 \%)$, nodularities (28\%), and polyps (28\%). It was reported in a Japanese study of 144 patients with non-EoE EGIDs that erosions (43\%), edema $(42 \%)$, and erythemas (38\%) were the common endoscopic findings in the gastrointestinal tract [3]. Zhang et al. examined 42 patients with EGE and reported that of the endoscopic abnormalities in the gastrointestinal tract observed, including mucosal hyperemia, area of roughening, scattered or widespread erythema, erosions, superficial ulcers, and nodularity, erythema was the most common [18]. In a multicenter retrospective cohort study by Pesek et al., endoscopic findings in patients with non-EoE EGIDs were analyzed. They examined 376 patients with non-EoE EGIDs, which included 317 children, and EG was found in 142 patients and EGE in 123 patients. Gastric endoscopy performed on the 265 patients with EG or EGE revealed normal healthy mucosa in 169 patients (64\%), erythema in $52(22 \%)$, nodularity in $23(9 \%)$, and ulcer in $26(10 \%)$ [19]. The differences and similarities between our study and other studies may be due to race (Japanese/Asians or mostly Whites), age (adults only or predominantly children), and methodology (detailed analysis of endoscopic images or analysis of endoscopic records).

Erythema is commonly observed in several conditions and diseases. The Kyoto classification of gastritis [13-15] classifies erythema as diffuse redness, map-like redness, red streaks, patchy redness, and spotty redness. Erythema is affected by $\mathrm{H}$. pylori infection status, which can be positive, negative, or posteradication, but red streaks and patchy redness are observed irrespective of $H$. pylori infection status [13-15]. Although Goto et al. reported that red streaks in patients with EGE completely disappeared after corticosteroid treatment [20], the erythema observed in this study may be non-specific as two patients were H. pylori-positive and 3 patients had post-eradication $\mathrm{H}$. pylori infection status. In this study, diffuse redness and map-like redness were not observed, but localized diffuse redness, which represent strong redness in a restricted area and is different from the erythema described in the Kyoto classification, was observed in two patients. It is unclear whether localized diffuse redness is associated with EG and EGE; therefore, further accumulation of cases is necessary.

Several types of gastric ulcers were identified, and the SMTlike deep large ulcers found in 3 patients were different from H. pylori-positive peptic ulcer disease and non-steroidal anti-inflammatory drug (NSAID)-induced ulcers. Malignancies such as cancers and lymphomas were initially suspected in these patients before the final diagnosis was made. Similar SMT-like deep ulcers were described in previous case reports [21-23]. Multiple erosions are non-specific as they have several etiologies including stress, alcohol, and drugs such as NSAIDs.

Endoscopy revealed relative unique findings such as antral Penthorum-like appearances, multiple white granular elevations, muskmelon-like appearances, cracks, and antral rings. Of the three patients with antral Penthorum-like appearances, one patient had nodules of the relatively flat type in lines that resemble the bamboo join-like appearance of Crohn's disease $[24,25]$, but their locations are different (antrum versus upper gastric body). Endoscopic findings similar to antral Penthorumlike appearance [26] and bamboo joint-like appearance [27] have been identified in case reports. Discolorations had two patterns, namely the multiple patchy type and muskmelon-like appearances. Discoloration and white granular elevations may represent eosinophil accumulations like white exudates in EoE. A few studies have reported cracked mucosa in patients with EGE $[26,28]$. Magnifying narrow-band image endoscopy showed that cracks were defects of surface mucosal epithelium. Some patients had cracks in a linear pattern on the lesser curvature that resemble the linear furrows in EoE [11]. In a study by Miyamoto et al. [16], cracks were more frequently observed in chronic PPI users (24.4\%) than in controls (3.7\%). Further, $28 \%$ of the study patients had cracks and none had received PPIs, suggesting the high prevalence of cracks in patients with EG and EGE. In addition, cracks in PPI users are observed in the gastric body; however, cracks in 3 of 5 patients were located in the antrum. In one patient, the antral ring was similar to the rings in EoE [11]. These results suggest that some endo- 
scopic findings may be similar to those found in EoE. However, the pathogenesis of these unique endoscopic findings is unknown.

It is unclear whether endoscopic findings observed in this study are specific for EGIDs or associated with disease activity of EGIDs. All endoscopic findings in this study were obtained from patients with confirmed gastric eosinophilia, suggesting a histological active phase. Most endoscopic findings were improved by the treatment, but some findings, especially cracks, did not. This suggests that cracks might not be related to disease activity of EGIDs. This is further supported by the low positive rate of gastric eosinophilia in target biopsies of craks. The difference in treatment efficacy on improvement of endoscopic findings is discussed. First, refractory cases were included against several treatments. Second, doses or duration of the treatment might insufficiently improve endoscopic findings. Third, the treatment efficacy based on endoscopic findings were categorized into complete remission, remission, and no change/worsening. However, a detailed definition of endoscopic evaluation at post-treatment has not yet been established. Thus, future prospective studies are necessary.

No association was observed between the endoscopic findings and gastric eosinophilic infiltration, and positive rate of gastric eosinophilia was similar between target and random biopsies. In this study, the mean gastric eosinophil count was $82 \mathrm{eos} / \mathrm{hpf}$, which is similar to that reported in the study by Peresk et al. (mean peak number: 78-87 eos/hpf) [19]. However, in the study by Peresk et al., gastric eosinophilia was reported to be mostly found in gastric mucosa with normal appearance. The exact reasons for the common finding of normal features on endoscopy in western countries are unclear; however, this highlights the need for multiple biopsies when diagnosing patients refractory to standard treatment and patients suspected of having EGIDs.

This study has some limitations. First, this study was a retrospective single-center study with a small sample size due to the rarity of EG and EGE, but this is the first study to perform a detailed analysis of endoscopic gastric images of patients with EG or EGE. Second, we diagnosed EG and EGE based on guidelines from the Japanese Ministry of Health, Labor and Welfare [12], but the diagnostic criteria are difficult to define and have not yet been established internationally $[9,10]$ since Talley first described the concept of EGE in 1990 [29].

\section{Conclusion}

In conclusion, various endoscopic findings of gastric lesions were observed in patients with EG or EGE. Of these, SMT-like ulcers, antral Penthorum-like appearances, muskmelon-like appearances, and cracks might be associated with EGIDs. Further studies that incorporate detailed pathological examination and image-enhanced endoscopy should be conducted in the future.

\section{Acknowledgments}

The project by F-Study group was aimed at identifying novel endoscopic findings of the gastrointestinal tract at the Department of Gastroenterology, Osaka City University Graduate School of Medicine. This work was supported by a Grant-in-Aid for Scientific Research from the Ministry of Education, Culture, Sports, Science, and Technology in Japan (18K07917).

\section{Competing interests}

The authors declare that they have no conflict of interest.

\section{References}

[1] Ishihara S, Kinoshita Y, Schoepfer A. Eosinophilic esophagitis, eosinophilic gastroenteritis, and eosinophilic colitis: common mechanisms and differences between East and West. Inflamm Intest Dis 2016; 1: 63-69

[2] Gonsalves N. Eosinophilic gastrointestinal disorders. Clin Rev Allergy Immunol 2019; 57: 272-285

[3] Kinoshita $\mathrm{Y}$, Furuta $\mathrm{K}$, Ishimaura $\mathrm{N}$ et al. Clinical characteristics of Japanese patients with eosinophilic esophagitis and eosinophilic gastroenteritis. J Gastroenterol 2013; 48: 333-339

[4] Dellon ES, Hirano I. epidemiology and natural history of eosinophilic esophagitis. Gastroenterology 2018; 154: 319-332

[5] Kinoshita $\mathrm{Y}$, Ishimura N, Oshima $\mathrm{N}$ et al. Systematic review: Eosinophilic esophagitis in Asian countries. World J Gastroenterol 2015; 21: 8433-8440

[6] Jensen ET, Martin CF, Kappelman MD et al. Prevalence of eosinophilic gastritis, gastroenteritis, and colitis: estimates from a national administrative database. J Pediatr Gastroenterol Nutr 2016; 62: 36-42

[7] Dellon ES, Liacouras CA, Molina-Infante J et al. Updated International Consensus Diagnostic Criteria for Eosinophilic Esophagitis: Proceedings of the AGREE Conference. Gastroenterology 2018; 155: 10221033

[8] Lucendo AJ, Molina-Infante J, Arias Á et al. Guidelines on eosinophilic esophagitis: evidence-based statements and recommendations for diagnosis and management in children and adults. United Eur Gastroenterol J 2017; 5: 335-358

[9] Kinoshita Y, Ishihara S. Eosinophilic gastroenteritis: epidemiology, diagnosis, and treatment. Curr Opin Allergy Clin Immunol 2020; 20: 311-315

[10] Uppal V, Kreiger P, Kutsch E. eosinophilic gastroenteritis and colitis: a comprehensive review. Clin Rev Allergy Immunol 2016; 50: 175-188

[11] Hirano I, Moy N, Heckman MG et al. Endoscopic assessment of the oesophageal features of eosinophilic oesophagitis: validation of a novel classification and grading system. Gut 2013; 62: 489-495

[12] Japan Intractable Diseases Information Center. Eosinophilic GastroIntestinal Disorder. 2020: https://wwwnanbyouorjp/entry/3935

[13] Haruma K. Kyoto Classification of Gastritis. Tokyo, Japan: Nihon Medical Center; 2018

[14] Kamada T, Haruma K, Inoue K et al. Helicobacter pylori infection and endoscopic gastritis -Kyoto classification of gastritis. Nihon Shokakibyo Gakkai Zasshi 2015; 112: 982-993

[15] Toyoshima O, Nishizawa T, Koike K. Endoscopic Kyoto classification of Helicobacter pylori infection and gastric cancer risk diagnosis. World J Gastroenterol 2020; 26: 466-477 
[16] Miyamoto S, Kato M, Tsuda M et al. Gastric mucosal cracked and cobblestone-like changes resulting from proton pump inhibitor use. Dig Endosc 2017; 29: 307-313

[17] Kanda Y. Investigation of the freely available easy-to-use software 'EZR' for medical statistics. Bone Marrow Transplant 2013; 48: 452458

[18] Zhang L, Duan L, Ding S et al. Eosinophilic gastroenteritis: clinical manifestations and morphological characteristics, a retrospective study of 42 patients. Scand J Gastroenterol 2011; 46: 1074-1080

[19] Pesek RD, Reed CC, Collins MH et al. Association between endoscopic and histologic findings in a multicenter retrospective cohort of patients with non-esophageal eosinophilic gastrointestinal disorders. Dig Dis Sci 2020; 65: 2024-2035. doi:10.1007/s10620-019-05961-4 [Epub ahead of print]

[20] Goto A, Nishikawa J, Okamoto T et al. eosinophilic gastroenteritis presenting with red streaking. Intern Med 2017; 56: 571-572

[21] Kristopaitis T, Neghme C, Yong SL et al. Giant antral ulcer: A rare presentation of eosinophilic gastroenteritis-case report and review of the literature. Am J Gastroenterol 1997; 92: 1205-1208

[22] Katsumi N, Yamaguchi Y, Yamato T et al. Multiple ulcerative lesions of the stomach: a rare case of eosinophilic gastroenteritis. Gastrointest Endosc 2002; 56: 762-764
[23] Tomizawa T, Kawamura O, Kusano M. A case of proton pump inhibitor-resistant multiple gastric ulcers caused by eosinophilic gastroenteritis. Clin Gastroenterol Hepatol 2015; 13: A23-A24

[24] Yokota K, Saito Y, Einami K et al. A bamboo joint-like appearance of the gastric body and cardia: possible association with Crohn's disease. Gastrointest Endosc 1997; 46: 268-272

[25] Fujiya M, Sakatani A, Dokoshi T et al. A bamboo joint-like appearance is a characteristic finding in the upper gastrointestinal tract of Crohn's disease patients: a case-control study. Medicine (Baltimore) 2015; 94: e1500

[26] Hiremath G, Pavis CM, Hicks J et al. Unusual endoscopic presentation of eosinophilic gastroenteropathy. Ann Gastroenterol 2015; 28: 145

[27] Sato H, Honma T, Owaki T et al. Clinical and pathological profile of eosinophilic gastroenteritis. Eur J Gastroenterol Hepatol 2019; 31: 157-162

[28] Nakamura A, Iwaya Y, Okamura T et al. Eosinophilic gastroenteritis complicated with Helicobacter pylori infection unresponsive to eradication therapy. Intern Med 2014; 53: 2061-2065

[29] Talley N], Shorter RG, Phillips SF et al. Eosinophilic gastroenteritis: a clinicopathological study of patients with disease of the mucosa, muscle layer, and subserosal tissues. Gut 1990; 31: 54-58 